\title{
A Rare Case of Mosaic Unbalanced Non-Robertsonian Translocation Involving Chromosomes 15 and 22 with Congenital Abnormalities in Monozygotic Twins
}

\author{
Emine I. Atli Engin Atli Sinem Yalcintepe Hakan Gurkan
}

Department of Medical Genetics, Faculty of Medicine, Trakya University, Edirne, Turkey

\section{Established Facts}

- Precise genetic diagnosis is crucial to delineate genotype/phenotype correlation and provide adequate genetic counseling for families of dysmorphic patients.

\section{Novel Insights}

- The clinical findings observed in patients may not always fully match the given syndromes. Therefore, both cytogenetic and molecular analyses are key diagnostic tools and should be systematically performed.

- Mosaicism ratio may lead to variable expressivity of the clinical phenotype.

\section{Keywords}

Array CGH · Autism · Chromosomal microarray analysis ·

Copy number variations · Deletion syndrome ·

Dysmorphology · FISH · Genotype-phenotype correlation ·

Mosaicism

\section{Abstract}

Balanced de novo non-robertsonian translocations (nonRTs), which involve acrocentric chromosomes, are rare findings in clinical cytogenetics and may be associated with an abnormal phenotype. These translocations, detected by conventional karyotyping, are found in approximately $1: 1,000$ neonates. In most of these cases, one of the parents carries the same translocation. In this study, we report a rare non-RT involving chromosomes 15 and 22 defined as
45,XX,-22, der(15; 22)t(15; 22)/46,XX,der(15)t(15; 22), der(22). To our knowledge, this is the first report of a non-RT $t(15 ; 22)$ with these breakpoints.

(c) 2019 S. Karger AG, Basel

Robertsonian translocations (RTs) are the most common balanced structural chromosomal anomalies involving acrocentric chromosomes in the population (usually nonhomologous and rarely homologous) with an incidence of 1.23/1,000 live births. In RT, the pericentric regions of the 2 acrocentric chromosomes merge with a single centromere or 2 centromers. This stable karyotype contains 45 chromosomes as a result of fusion of the long arms of 2 displaced acrocentric chromosomes [Groupe de Cytogénéticiens Français, 1989; Nielsen and Wohlert, 1991]. Balanced de novo non-robertsonian transloca-

\section{KARGER}

(c) 2019 S. Karger AG, Basel

E-Mail karger@karger.com

www.karger.com/msy
Emine Ikbal Atli

Department of Medical Genetics, Faculty of Medicine Trakya University

Balkan Campus, Highway D100, Edirne 22030 (Turkey)

E-Mail eikbalatli@ trakya.edu.tr 
Fig. 1. Pedigree of the patients.

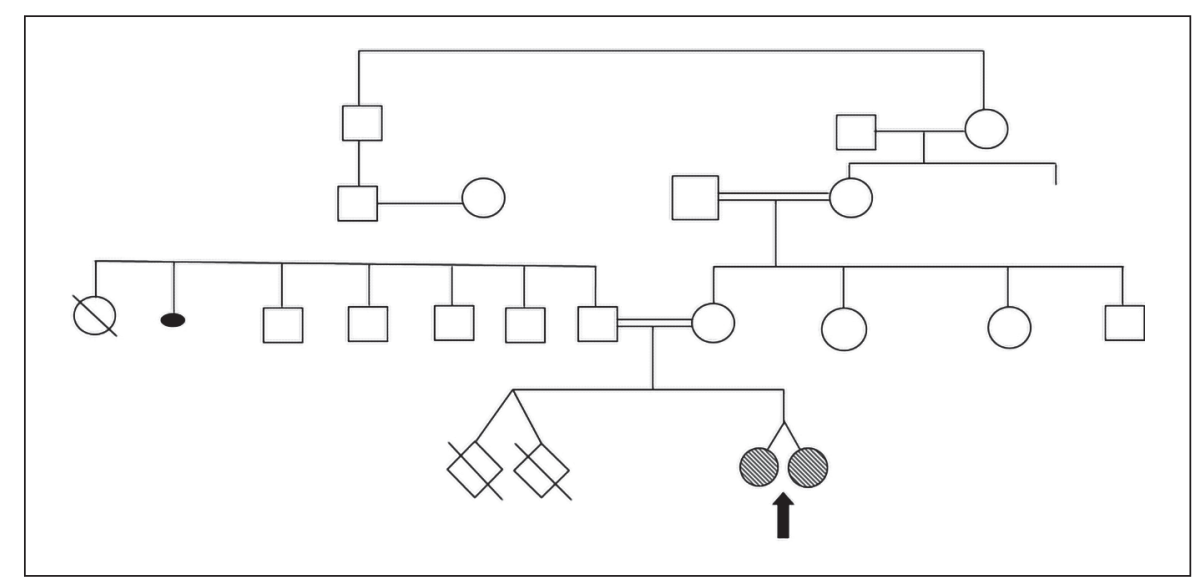

tions (non-RTs), which involve acrocentric chromosomes, are rare findings in clinical cytogenetics and may be associated with abnormal phenotypes. Data in the literature emphasize that most of the balanced de novo nonRTs are of paternal origin, but predominance does not seem as prominent as previously thought, and paternity age is not necessarily seen as an important contributing factor [Höckner et al., 2012]. Balanced non-RTs detected by conventional karyotyping are found in approximately 1:1,000 neonates. In most of these cases, one of the parents carries the same translocation [Gardner and Sutherland, 2003].

In this study, we report a rare non-RT case involving chromosomes 15 and 22 defined as 45,XX,-22, der $(15 ; 22)$ $\mathrm{t}(15 ; 22) / 46, \mathrm{XX}, \operatorname{der}(15) \mathrm{t}(15 ; 22), \operatorname{der}(22)$. To our knowledge, this is the first report of a non-RT $t(15 ; 22)$ with these break points.

\section{Case Reports}

A 14-year-old girl was referred to the Trakya University Medical Genetics Department because of autism spectrum disorder with behavior and anxiety disorders. She has been in a follow-up process in the child psychiatry department of the university. Abasia and speech disorder were detected in the patient. The previous pregnancy of the mother also was a twin pregnancy but resulted in spontaneous abortion after the 5th month (Fig.1). The recent prenatal period was considered having a low risk. Ultrasonography scans were considered normal. Birth was performed by cesarean section in the 7th month. The patient's birth weight was 1,600 $\mathrm{g}$ ( $<3$ rd percentile); her twin sister (identical twin) weighed 2,400 $\mathrm{g}$ (3rd percentile) and required an incubator for 16 days. In our case, congenital heart disease was diagnosed (atrial septal defect). Therefore, angiography was not done before she was 6 years old. Dysmorphic findings of the patient such as a narrow forehead, downslanting palpebral fissures, short filtrum, slight microti, distorted tooth structure, long big toe, small foot structure, and a prominent nasal root/bridge were found (Fig. 2). Age at menarche was 12 years. Her twin sister had suspected autism spectrum disorder with behavior and anxiety disorders as well as stuttering. A bowel anomaly was detected at birth. She underwent surgery 3 times, and vomiting was observed until the age of 8 years. The girl was able to walk at 6.5 years. No speech was observed. Short and narrow skull shape in this twin sibling, mild synophrys in the periocular region, prominent nasal root, bulbous nose, thin upper lip, retrognathia, microti, small hand structure, and genitalia are consistent with Prader stage 5 (Fig. 3). Age at menarche was 10 years. As a result of clinical findings, chromosome analysis, array CGH, and fragile $\mathrm{X}$ syndrome screening were performed on both sisters.

\section{Materials and Methods}

For conventional karyotyping, standard 72-h lymphocyte culture and harvesting was performed from the patient's peripheral blood sample by Leishmann-Pancreatin banding. Analysis results were reported according to the International System for Human Cytogenomic Nomenclature [ISCN 2016]. For each sample, 42 metaphase plates were examined.

Slides were prepared using peripheral blood specimens and FISH was performed using the manufacturer's recommendations (Cytocell Technologies, Cambridge, UK). A total of 200 interphase cells were analyzed for each sample with images captured and stored using Applied Imaging/Cytovision system (Fig. 4). Final results were reported using the cutoffs established in the laboratory for each of the probes tested.

Two milliliters of peripheral venous blood samples of the patients were collected in EDTA tubes. Genomic DNA was extracted according to the protocol of the kit used (EZ1 Advanced Instruments, Qiagen, Hilden, Germany). The concentration and purity values of the DNA samples were measured on the NanoDrop device (Nanodrop 2000C, Thermo Fisher Scientific, Waltham, MA, USA). The DNA obtained from peripheral blood samples were then investigated for genetic aberrations by array CGH using the Cytosure 4x180K oligonucleotide array (Agilent Technologies, Inc., Santa Clara, CA, USA). For each sample, $20.2 \mu \mathrm{L}$ genomic DNA was used. The CytoSure Genomic DNA aCGH labeling kit (Agilent) was used to label the reference and patients DNA with Cy3 and Cy5, respectively. The labeled targets were then purified 

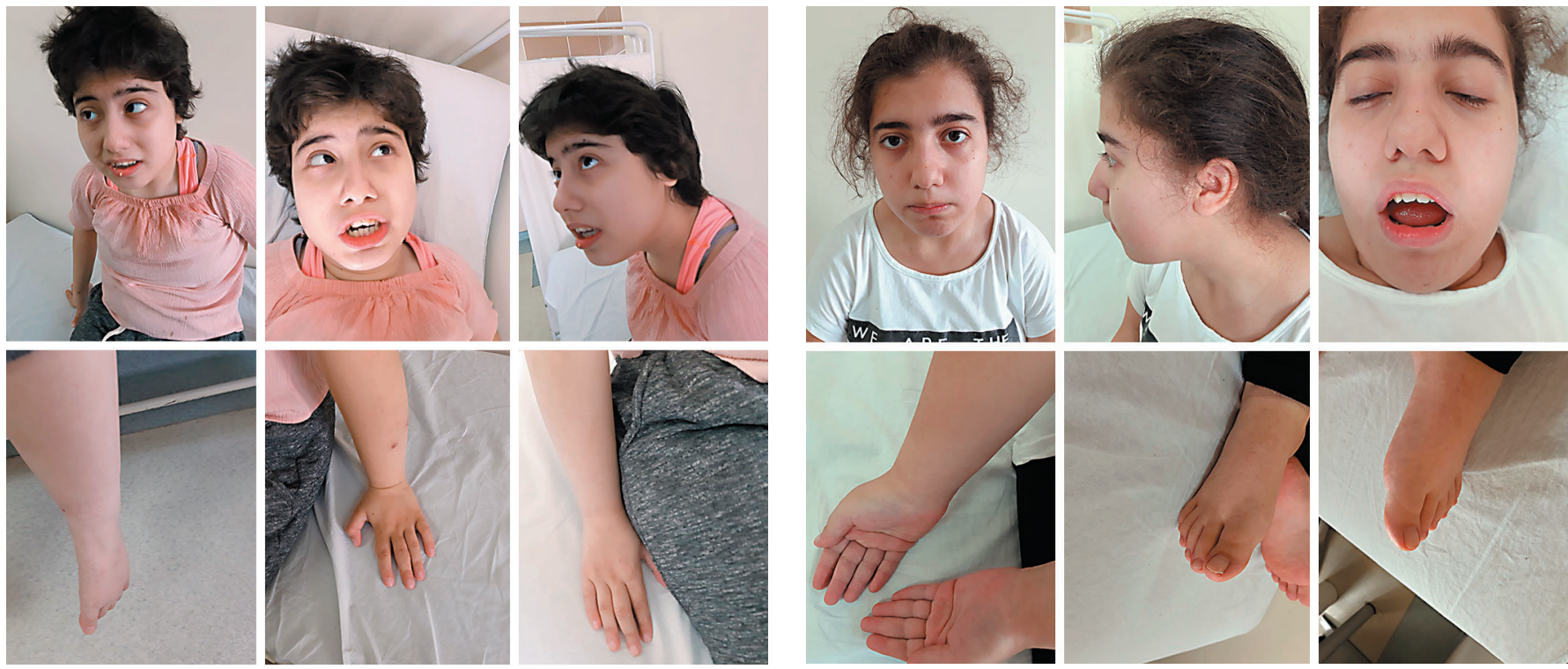

Fig. 2. Phenotypic findings of twin I.

Fig. 3. Phenotypic findings of twin II.
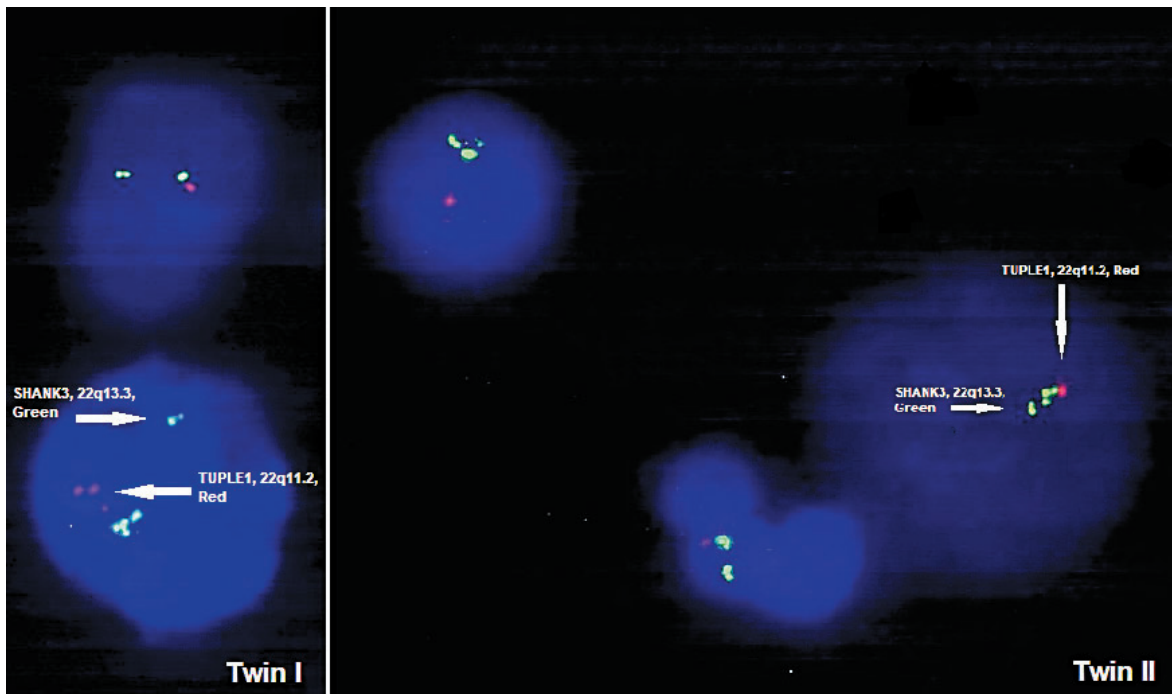

Fig. 4. FISH analysis of twin I and II. The TUPLE1 (22q11) probe is labeled in red and covers most of the HIRA (former TUPLE1) gene. The N85A3 probe, labeled in green, is located within 22q13.3 and covers the telomeric end of the SHANK3 gene. Green signals appear on the $\mathrm{q}$ arm of a normal 22 and also on a translocated 22 chromosome. The red signal is only visible on chromosome 22 . The other 22q11 was deleted and no red signal was observed.

according to the manufacturer's protocol with a minimum labeling efficiency of $250 \mathrm{pmol} / \mu \mathrm{g}$ DNA for Cy3 and $200 \mathrm{pmol} / \mu \mathrm{g}$ DNA for Cy5. Following, the purified $\mathrm{Cy} 3$ and $\mathrm{Cy} 5$ labeled genomic DNA was mixed with human Cot 1 DNA, blocking agent, and hybridization buffer, and then was denatured and incubated at $37^{\circ} \mathrm{C}$ for $30 \mathrm{~min}$. A total of $100 \mu \mathrm{L}$ sample mix was then pipetted into the hybridization chamber containing the microarray and placed in a rotisserie hybridization oven at $65^{\circ} \mathrm{C}$ for $40 \mathrm{~h}$. Subsequent to hybridization, the slides were washed with 2 wash buffers: wash buffer 1 for $5 \mathrm{~min}$ at room temperature and wash buffer 2 for $1 \mathrm{~min}$ at $37^{\circ} \mathrm{C}$. The slides were then scanned using the Agilent DNA scanner to produce the raw image data. This data was further processed using the Agilent Feature Extraction software (Feature Extraction 9.1, CGH Analytics 4.5) and the results analyzed with the Cytosure
Interpret software v3.4.2 (Agilent). A consensus cutoff for recording an alteration was a copy number variation involving at least 3 consecutive oligonucleotides, presenting an abnormal ratio higher than +0.58 or lower than -0.75 . An in silico analysis of the unbalanced regions was done using the UCSC Genome Browser (https:// genome.ucsc.edu/), DECIPHER (https://decipher.sanger.ac.uk/), DGV (http://dgv.tcag.ca/dgv/app/home), and OMIM (https:// omim.org/) databases.

Bisulfite DNA was extracted according to the manufacturer's instructions (EpiTect Bisulfite convention Kit, Qiagen). As an alternative method for the diagnosis of Fragile X syndrome, fluorescent methylation-specific PCR was performed on AB 3130×l (Avant System, Applied Biosystems, Foster City, CA, USA) and analyzed with the Gene Mapper v5.0 software program. 


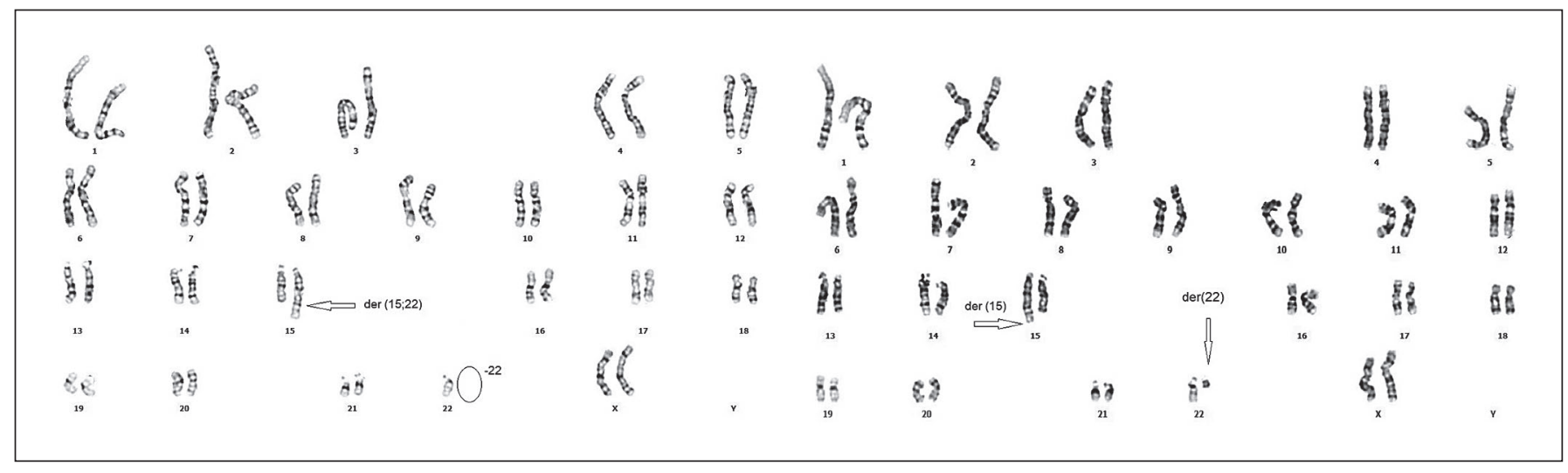

Fig. 5. Twin I karyotype. 45,XX,-22, $\operatorname{der}(15 ; 22) t(15 ; 22)[23 / 42] / 46, X X, \operatorname{der}(15) t(15 ; 22), \operatorname{der}(22)[19 / 42]$.

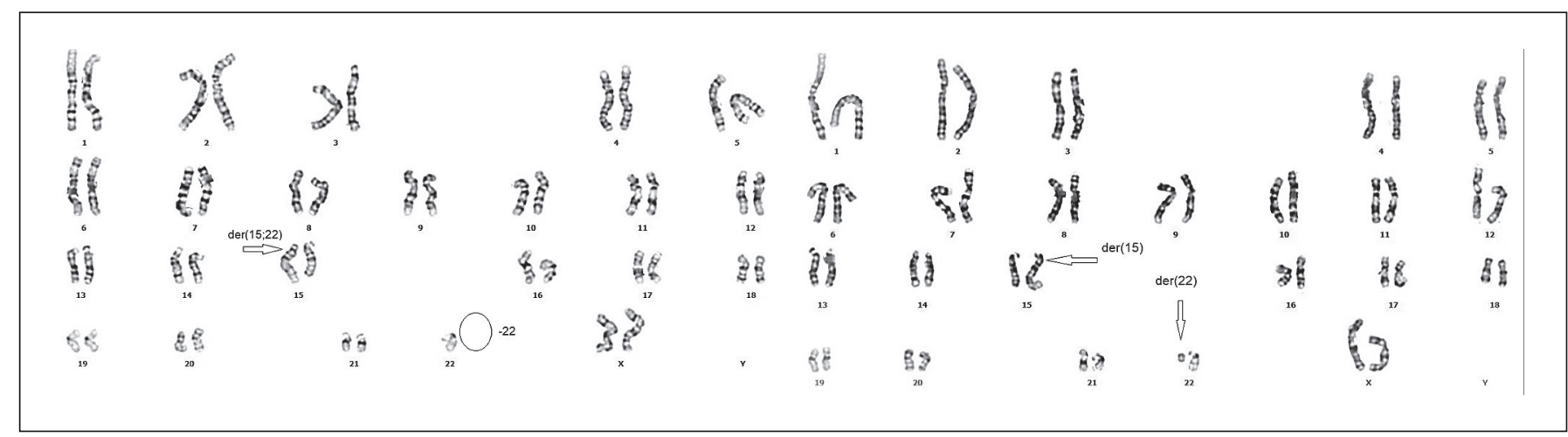

Fig. 6. Twin II karyotype. 45,XX,-22, der(15;22)t(15;22)[38/54]/46,XX,der(15)t(15;22), der(22)[16/54].

\section{Results}

Mosaicism was detected in the analyzed metaphase plates: a 45,XX,-22,der(15;22)t(15;22)(q26.3;q11.1) karyotype was determined in 23 metaphases and a 46,XX,der(15)t(15;22)(q26.3;q11.1),der(22) karyotype in 19 metaphases (Fig. 5). In the patient's twin sister a $45, \mathrm{XX},-22, \operatorname{der}(15 ; 22) \mathrm{t}(15 ; 22)(\mathrm{q} 26.3 ; \mathrm{q} 11.1)$ karyotype was found in 38 metaphase plates and a 46,XX,der(15) $\mathrm{t}(15 ; 22)$ (q26.3;q11.1),der(22) karyotype in 16 metaphase plates (Fig. 6). A translocation involving chromosomes 15 and 22 was detected in the 45-chromosome karyotype. Additional material was observed on the long arm of the chromosome 15 and one chromosome 22 was missing. Derivative (15) replaces a normal chromosome 15, and one of the homologous chromosomes 22 is lost. In this way, 23 metaphases were observed. Metaphases with 46

Mosaic Unbalanced Non-Robertsonian

Translocation in Monozygotic Twins chromosomes were found to have 2 derivative chromosomes. The karyotypes of the parents were normal.

According to the patient's array-CGH result, there is a copy number loss in the chromosome region of 22q11.1q11.21 (2,574 kb in size) (Fig. 7). Balanced rearrangements and mosaicism could not be detected because of the limitations of the array-CGH method. The deleted chromosome region in the patient contains the velocardiofacial syndrome (VCFS; OMIM 192430) region and led to the diagnosis of VCFS.

Since the phenotypic manifestations of VCFS are pleiotropic, a differential diagnosis was performed to consider various diseases. VCFS is a multisystemic syndrome and is difficult to detect; at least 30 different symptoms are associated with this $22 \mathrm{q} 11$ deletion, and symptoms vary from child to child (https://www.genome. gov/25521139). 


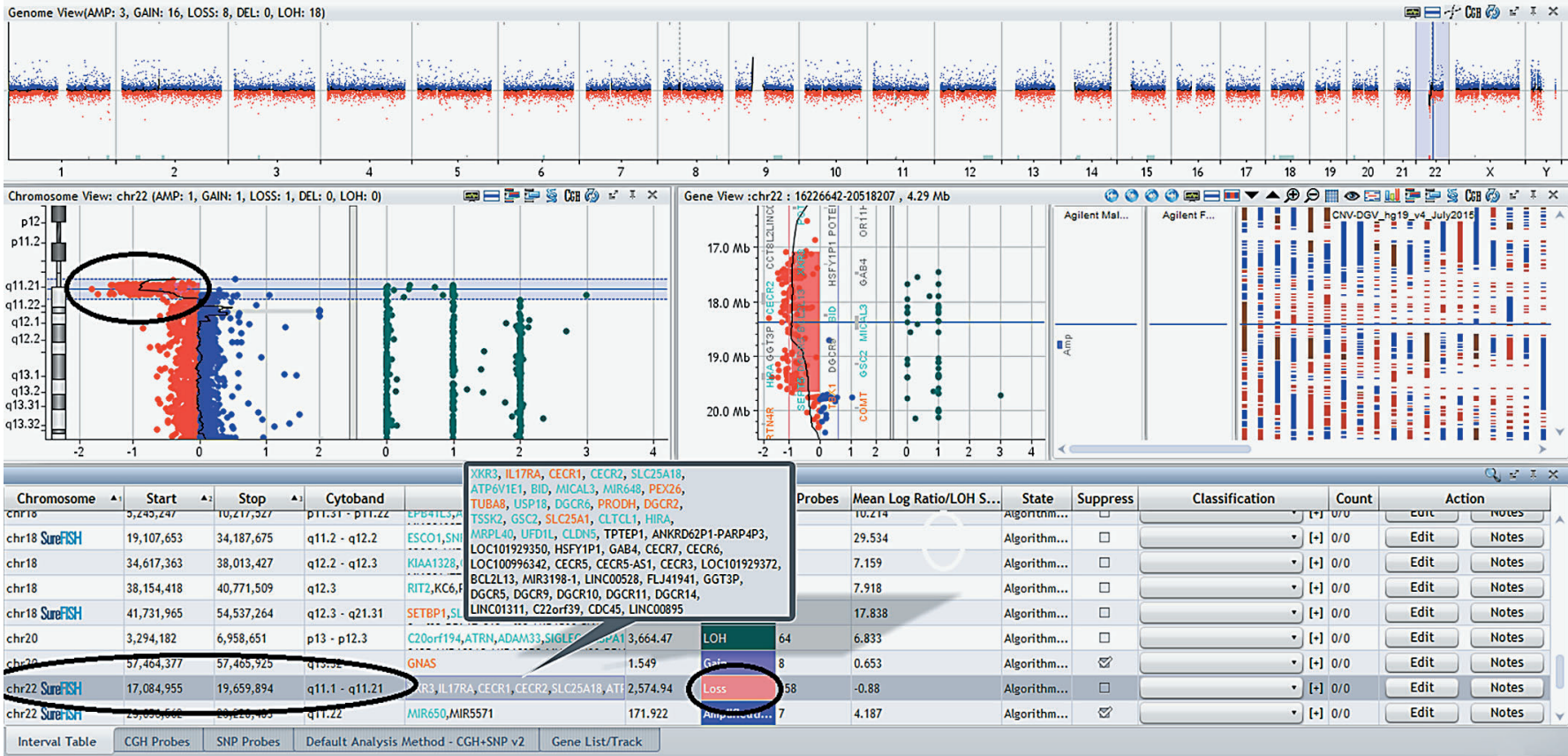

b

Genome View(AMP: 3, GAIN: 12, LOSS: 7, DEL: 1, LOH: 18) Chromosome View: chr22 (AMP: 1, GAlN: 1, LOSS: 1, DEL: 0, LOH: 0 )

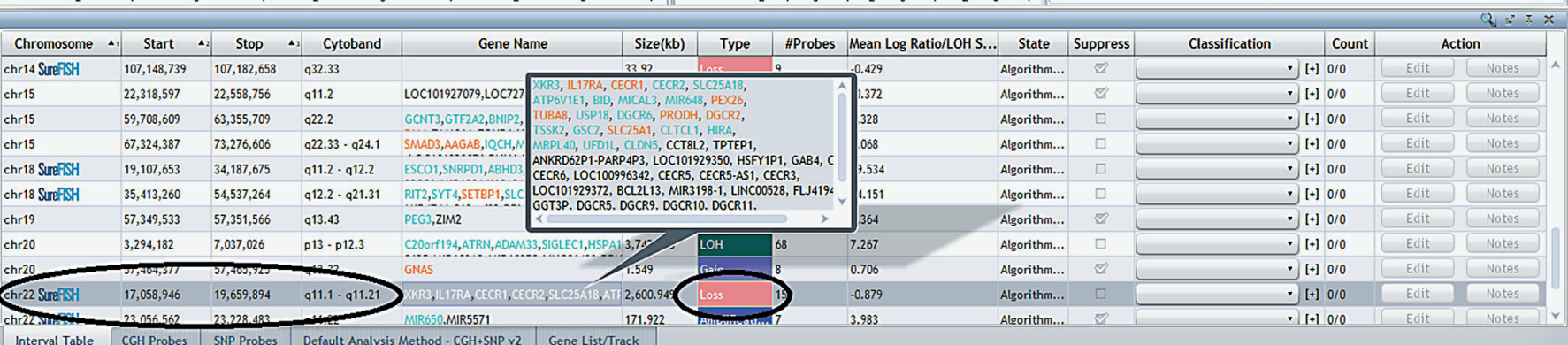

Fig. 7. Array-CGH analysis of twin I (a) and twin II (b).

\section{Discussion}

VCFS is caused by a microdeletion in chromosome $22 \mathrm{q} 11.2$ and is the most common type of contiguous gene syndrome in humans. VCFS occurs 1/4,000-7,000 births
[Gothelf et al., 2009]. The syndrome has been previously described by many researchers and given many different names including, Shprintzen syndrome, DiGeorge syndrome (DGS), DiGeorge gene sequence, CATCH22, 22q11.2 deletion syndrome, Cayler syndrome, and 
conotruncal anomaly face syndrome. VCFS is the fourth most common congenital anomaly in the world, and many healthcare professionals now refer to VCFS as $22 \mathrm{q} 11.2$ deletion syndrome. The deleted region of the chromosome contains information about the development of organs in the third and fourth pharyngeal sacs during the 12th week of pregnancy. VCFS is a multiple malformation syndrome characterized by highly variable clinical features including cleft palate, cardiac anomalies, atypical facial development, and cognitive and neuropsychological difficulties [Shprintzen et al., 1978; Sandrin-Garcia et al., 2007].

In the first broad study of VCFS, 156 cases with a localized deletion at $22 \mathrm{q} 11$ were evaluated, and no relationship was found between the size of the deletion and the phenotype [Carlson et al., 1997].

There are many different translocations between chromosome 22 and other chromosomes. This is due to the presence of a region in $22 \mathrm{q} 11.2$ that contains 8 chromosome-specific low copy repeats, a highly conserved DNA sequence $(>96 \%)$ that induces nonallelic homologous recombination [Gollo Dantas et al., 2016].

In another patient with a $46, \mathrm{XY},-15,+$ der $(22), \mathrm{t}(15 ; 22)$ (q13;q11)pat translocation, it was reported that the patient had typical clinical findings of the 15pterq13 (severe hypotonia and lethargy) deletion and also typical signs of 22q11qter duplication such as hypertelorism, downslanting small palpebral fissures, preauricular tags, and long philtrum [Van Hove et al., 1992]. In another case, frontonasal malformation and reciprocal $\mathrm{t}(15 ; 22)(\mathrm{q} 22 ; \mathrm{q} 13)$ translocation without 15 or 22 monosomy have been reported [Fryns et al., 1993]. The etiology of these cases coinciding with our case is very rare. To our knowledge, mosaic monosomy of chromosome 22 has been reported, which is associated with a unique facial appearance similar to DGS, with only 7 live births [Pinto-Escalante et al., 1998]. Herein, we describe the first case of twins with this de novo mosaic aberration in both individuals. The rates of metaphases analyzed in twin patients were $23 / 19$ and 38/16, respectively.

Mosaicism for structural chromosomal abnormalities is rare and can be difficult for genetic counseling, especially when detected prenatally. Our cases suggested that one of the parents might have gonadal mosaicism (GM), especially regarding the fact that the previous twin pregnancy resulted in a miscarriage. Unfortunately, no genetic analysis was performed on the abortion material to confirm this.

Identification and counseling of GM may be more problematic than being asymptomatic and cryptic in the GM carrier. There are 2 hypotheses regarding the presence of

Mosaic Unbalanced Non-Robertsonian

Translocation in Monozygotic Twins
GM discussed in the literature. The first is that the mutation occurs in a germ cell that continues to divide (limited mosaicism to germ cells). The second is that the mutation occurs very early in a somatic cell before it is separated into germinal cells and is therefore present in both somatic and germinal cells. In a previous study, it was reported that there is a strong female predominance among the carriers of mosaicism for changes with pericentromeric breaks, indicating female-specific instability in early human embryos [Zlotogora, 1998; Kovaleva, 2005].

Translocation $\mathrm{t}(3 ; 22)(\mathrm{p} 25 ; \mathrm{q} 11)$ and translocation $\mathrm{t}(6$; 22)(p25.3;q11.21) have been reported in DGS/VCFS cases born with in vitro fertilization [Faed et al., 1987; Gollo Dantas, et al., 2016]. In these patients, loss of the proximal $22 \mathrm{q}$ region results in $22 \mathrm{q} 11.2$ deletion syndrome, usually associated with the monosomy of chromosome 22. The rearrangements may be due to manipulation of the embryo or a sporadic event that is not related to in vitro fertilization.

Less than $1 \%$ of the $22 \mathrm{q} 11$ deletions are the result of an unbalanced translocation including chromosome 22 and another chromosome. In our case, the translocation was detected in chromosomes 15 and 22. The breakpoint in chromosome 22 is in the $22 \mathrm{q} 11$ band, the critical region for DGS 1, and the pericentromeric region of chromosome 22 was detected in only some of the metaphases. This cytogenetic aberration is associated with a phenotypic appearance suggesting VCFS. The family received genetic counseling including mode of transmission, and clinical and social aspects of this anomaly.

Approximately $93 \%$ of all the patients had $22 \mathrm{q} 11$ de novo deletions, while $7 \%$ of the patients inherited $22 \mathrm{q} 11$ deletions from a parent [McDonald-McGinn, et al., 2001]. Genetic counseling can be very difficult and complicated in our patient and similar cases. Chromosome 22q11band and chromosome 15q11/q13-band regions are considered unstable regions [Capra et al., 2013]. The genetic risk of a family with a congenital anomaly is present in each future pregnancy. Therefore, it was recommended that the mother should consider invasive prenatal diagnosis [Popovici, 2011].

In conclusion, this translocation in the 45-chromosome karyotype or the 46-chromosome karyotype is a rare event, and to our knowledge, translocations involving chromosomes $15 \mathrm{q}$ and $22 \mathrm{q}$ have previously been described, but in this study, we report the first case as mosaic in monozygotic twins, which could explain the phenotype of the patients. Precise genetic diagnosis is invaluable in order to provide adequate genetic counseling to the affected families. 


\section{Acknowledgment}

The authors would like to thank their colleagues from the Trakya University, School of Medicine, Department of Pediatrics (Edirne, Turkey) for their cooperation during the case evaluation.

\section{Statement of Ethics}

This study was approved by the Trakya University Faculty of Medicine Scientific Research Ethics Committee. Written informed consent was given to publish the medical information associated with the patients.

\section{Disclosure Statement}

The authors have no conflicts of interest to declare.

\section{Author Contributions}

E.I. Atli conceived the study, participated in its design, and drafted the manuscript. E. Atli carried out the molecular/cytogenetic studies. S. Yalcintepe performed all examinations. H. Gurkan conceived the study.

\section{References}

-Capra V, Mascelli S, Garrè ML, Nozza P, Vaccari $\mathrm{C}$, et al: Parental imbalances involving chromosomes $15 q$ and $22 q$ may predispose to the formation of de novo pathogenic microdeletions and microduplications in the offspring. PLoS One 8:e57910 (2013).

-Carlson C, Sirotkin H, Pandita R, Goldberg R, McKie J, et al: Molecular definition of 22q11 deletions in 151 velo-cardio-facial syndrome patients. Am J Hum Genet 61:620-629 (1997).

Faed MJ, Robertson J, Beck JS, Cater JI, Bose B, Madlom MM: Features of di George syndrome in a child with $45, \mathrm{XX},-3$,$22,+\operatorname{der}(3), \mathrm{t}(3 ; 22)(\mathrm{p} 25 ; \mathrm{q} 11) . \mathrm{J}$ Med Genet 24: 225-227 (1987).

Fryns JP, Kleczkowska A, van den Berghe H: Frontonasal malformation and reciprocal translocation $\mathrm{t}(15 ; 22)(\mathrm{q} 22 ; \mathrm{q} 13)$. Clin Genet 44:46-47 (1993).

Gardner RJM, Sutherland G: Chromosome Abnormalities and Genetic Counseling, 3rd ed, No. 46 (Oxford University Press, New York 2003).

Gollo Dantas A, Bortolai A, Moysés-Oliveira M, Takeno Herrero S, Azoubel Antunes A, et al: 22q11.2 deletion syndrome due to a translocation $\mathrm{t}(6 ; 22)$ in a patient conceived via in vitro fertilization. Mol Syndromol 6:242-247 (2016).
Gothelf D, Frisch A, Michaelovsky E, Weizman A, Shprintzen RJ: Velo-cardio-facial syndrome. J Ment Health Res Intellect Disabil 2:149-167 (2009).

Groupe de Cytogénéticiens Français: Robertsonian translocations and abnormal phenotypes. Groupe de Cytogénéticiens Français. Ann Genet 32:5-9 (1989).

Höckner M, Spreiz A, Frühmesser A, Tzschach A, Dufke A, et al: Parental origin of de novo cytogenetically balanced reciprocal non-Robertsonian translocations. Cytogenet Genome Res 136:242-245 (2012).

ISCN 2016: An International System for Human Cytogenomic Nomenclature; McGowan-Jordan J, Simons A, Schmid M (eds). Cytogenet Genome Res 149:1-140 (2016).

Kovaleva NV: Sex-specific chromosome instability in early human development. Am J Med Genet A 136A:401-413 (2005).

McDonald-McGinn DM, Tonnesen MK, Laufer-Cahana A, Finucane B, Driscoll DA, et al: Phenotype of the 22q11.2 deletion in individuals identified through an affected relative: cast a wide FISHing net! Genet Med 3:23-29 (2001).
Nielsen J, Wohlert M: Chromosome abnormalities found among 34,910 newborn children: results from a 13-year incidence study in Arhus, Denmark. Hum Genet 87:81-83 (1991).

Pinto-Escalante D, Ceballos-Quintal JM, Castillo-Zapata I, Canto-Herrera J: Full mosaic monosomy 22 in a child with DiGeorge syndrome facial appearance. Am J Med Genet 76: 150-153 (1998).

Popovici C: Profilaxia bolilor genetice, in Covic M, Ștefănescu D, Sandovici I (eds): Genetică Medicală, 2nd ed, pp 619-647 (Polirom Publishing House, Iași 2011).

-Sandrin-Garcia P, Richieri-Costa A, Tajara EH, Carvalho-Salles AB, Fett-Conte AC: Fluorescence in situ hybridization (FISH) screening for the 22q11.2 deletion in patients with clinical features of velocardiofacial syndrome but without cardiac anomalies. Genet Mol Biol 30:21-24 (2007).

-Shprintzen RJ, Goldberg RB, Lewin ML, Sidoti EJ, Berkman MD, et al: A new syndrome involving cleft palate, cardiac anomalies, typical facies, and learning disabilities: Velo-cardio-facial syndrome. Cleft Palate J 15:56-62 (1978).

-Van Hove JL, McConkie-Rosell A, Chen YT, Iafolla AK, Lanman JT Jr, et al: Unbalanced translocation $46, \mathrm{XY},-15,+\operatorname{der}(22) \mathrm{t}(15 ; 22)$ (q13;q11)pat: case report and review of the literature. Am J Med Genet 44:24-30 (1992).

Zlotogora J: Germ line mosaicism. Hum Genet 102:381-386 (1998). 